\title{
Influence of vertical movement in bathymetries and its influence on the measurement conditions.
}

\author{
[Raúl Pereda García, Julio Manuel de Luis Ruiz, Rubén Pérez Álvarez, Elena Castillo, Felipe Piña García]
}

\begin{abstract}
Bathymetries constitute a fundamental element of building objects settled on land submerged land: dikes, docks, underwater pipelines; in addition to dredges, volume of reservoir, etc. In civil engineering the bathymetry affect areas of small extent in which measures should be the most accurate as possible, influencing many factors in its precision. Among these factors can be distinguished: speed of sound in water, positioning system, vessel movements (pitch and roll) and vertical movement of the boat.

Vertical movement of the vessel is perhaps one of the least studied factors, but their influence on the accuracy of bathymetries could be very important depending on swell conditions and depth that exists at the time of measurement.

This paper defines a mathematical model based on measurements of bathymetry observed with GPS that lets you define wave conditions that found the boat according to its trajectory and speed. From this point, the article quantifies the effect of the vertical movement of the boat to correct its effect on the measured depths with an echo-sounder.
\end{abstract}

Finally, from the results it is possible to obtain a set of recommendations about operations to measure a bathymetry depending on the accuracy that you should obtain.

Keywords - Bathymetry, echo-sounder, Doppler effect, GPS.

Raúl Pereda García. Researcher ID: B-5240-2015

School of Civil Engineering / University of Cantabria / Spain

Julio Manuel de Luis Ruiz. Researcher ID: B-4956-2015

School of Mining and Energy Engineering / University of Cantabria / Spain

Rubén Pérez Álvarez. Researcher ID: B-8959-2015

School of Mining and Energy Engineering / University of Cantabria / Spain

Felipe Piña García. Researcher ID: B-5710-2015

School of Mining and Energy Engineering / University of Cantabria / Spain

Elena Castillo López. Researcher ID: M-1310-2015

School of Civil Engineering / University of Cantabria / Spain

\section{Introduction.}

Echo sounding bathymetries constitute a common technique for obtaining mapping underwater. Their applications in civil engineering are many (construction of dams, decks or submarine emissaries, dredging of harbours, determination of the volume of operating water reservoirs, definition of profiles for flood calculation in rivers, etc.) and they usually require detailed bathymetries which are constrained to small areas and they must be as accurate as possible.

In this sense, the accuracy is affected by a number of errors that occur during the different phases of modeling; [Maleika 2013]:

- Depth Reading error caused by measurement devices.

- Errors resulting from adopted certain operating parameters.

- Errors in determining the position.

- Errors that occur during the interpolation processes.

- Errors due to surface smoothing process.

- Errors that occur during the editing and publishing of cartography maps.

Among these, the errors due to navigation have been studied in depth and this is the case of pithc and roll; but less another ones like vertical movement due to the waves.

This article establishes a mathematical model to define this error solely on the data obtained by the element that allows to calculate the position (GPS) and, from it, set the corrections that could be applied and the conclusions that could give place.

\section{Instrumental y condicionantes.}

Vertical movement of the boat does not produce any deviation in the direction of the sounder but can equally influence the depth measurement.

It consists of the vertical movement of the boat due mainly to swell; and its consequence is a delay defined as the time that the wave of the sounder takes to travel the vertical movement that the vessel has made since it was issued until it is received its echo, that is, during depth measurement . Long period of vertical movements of the boat, such as tide, can be 
Proc. of the Fourth International Conference on Advances in Civil, Structural and Environmental Engineering - ACSEE 2016. Copyright ( $\odot$ Institute of Research Engineers and Doctors. All rights reserved.

ISBN: 978-1-63248-114-6 doi: 10.15224/ 978-1-63248-114-6-22

considered second order compared to small period because depth readings require very short times.

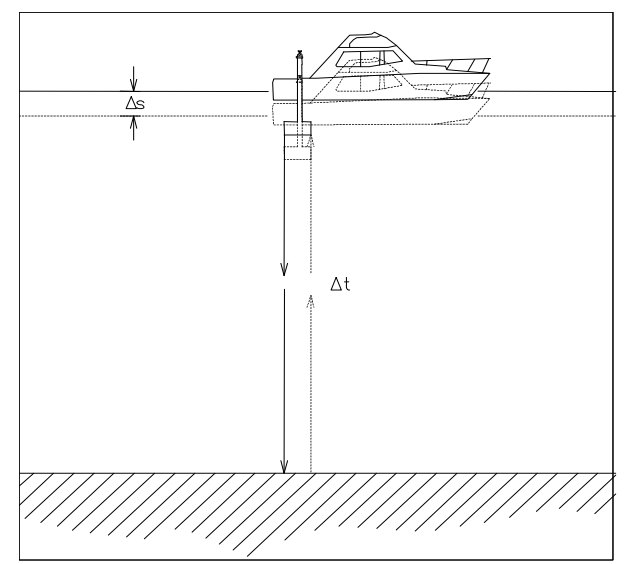

Figure 1. Vertical movement of the boat.

Thus, the vertical movement of the vessel, corresponding to the instant of measurement echo sounder with a duration $\Delta \mathrm{t}$, has a value $\Delta s$, and can be quantified as:

$$
\Delta t=\frac{p_{m}+\Delta s}{v_{c}} \approx \frac{p_{m}}{v_{c}}
$$

Being $v_{c}$ the velocity of the sound in the water (calibrated) and $p_{m}$ the measured depth.

The vertical offset of the vessel will be defined, therefore, from this movement that has been suffered in this interval of time.

Since the depth is calculated from the time that a pulse takes to reach the bottom and go back, this is going to be affected by an error caused by the displacement of the ship, and therefore, the sounder. To quantify the value of this correction to be applied to each depth readings must model the vertical movement of the boat. To this end, the proposed model only uses data obtained by the GPS which is placed on the sounder, allowing both the definition of the path and the movement to be studied.

\section{Mathematical model.}

\section{A. Mathematical model for vertical movement.}

Assuming that the main component of the vertical motion is due to swell, it is regarded as a periodic motion of a certain amplitude and period during the time that the depth reading is performed. Linear interpolation constitute an excessively rough approximation especially if the recording cadence is high, on the order of a second or higher, and generally comparable to the wave period.
The movements of pitch and roll can cause an increase or decrease of height sounder, especially if it is located on one side of the vessel, being included in the proposed model. However its amplitude is generally much smaller than the wave so, in principle, the proposed approach will be close to the conditions of movement of the water surface at each instant and in the direction of advance of the boat.

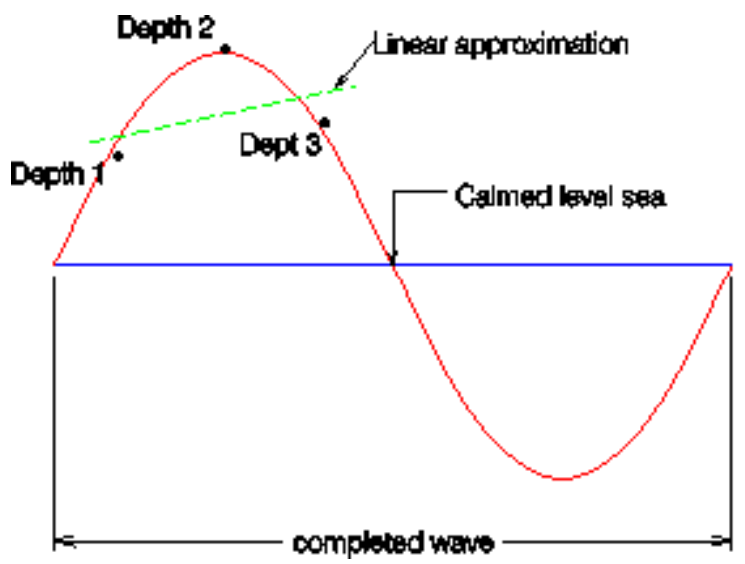

Figure 2. Mathematical model.

$$
z=F(t)=z_{i}+A \cdot \cos B t
$$

However, none of these factors is constant: the amplitude because swell conditions in the different work areas can vary significantly; and period depend on the angle that forms the vessel track with wave direction and the relative velocity between the ship and the swell, that is, because of Doppler effect.

Thus, you must determine the conditions of movement at all times, for which an approach is proposed by least squares discrete choosing the measures, the GPS receiver located on sounder, before and after which it is to be corrected. This way, these positions define the wave motion to be studied that we want to study.

In this way, if for three specific sound readings are known, the height of the GPS antenna receiver located on the echo sounder can be modeled, being the second the measure to be studied:

$$
\left(t_{1}, z_{1}\right) ;\left(t_{2}, z_{2}\right) ;\left(t_{3}, z_{3}\right)
$$

Applying the method of least squares to determine the parameters A, B should minimize the expression:

$$
M=\sum_{i=1}^{3}\left[z_{i}-\left(z_{1}+A \cdot \cos B t\right)\right]
$$


Proc. of the Fourth International Conference on Advances in Civil, Structural and Environmental Engineering - ACSEE 2016. Copyright (C) Institute of Research Engineers and Doctors. All rights reserved. ISBN: 978-1-63248-114-6 doi: 10.15224/ 978-1-63248-114-6-22

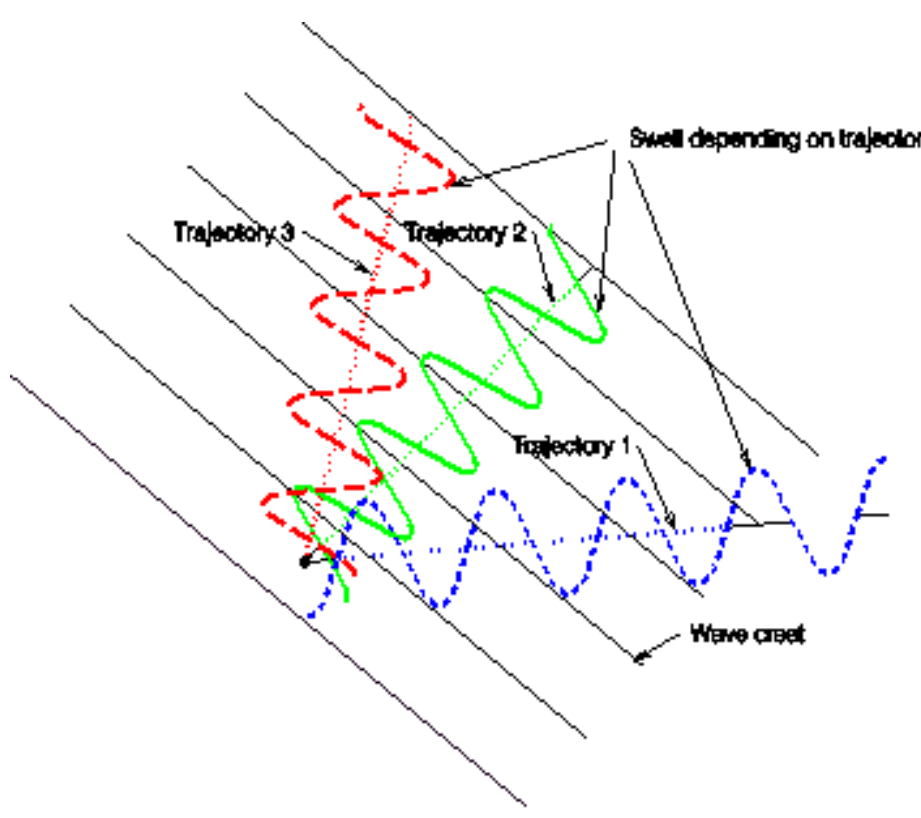

The values of the parameters A, B will be defined by solving the above equations system. Since it is a nonlinear system for resolution may apply some of the techniques of numerical analysis: Method of Newton, Quasi-Newton method, or another techniques. In this case we have chosen the technique known as Newton Method [R.L.BURDEN], which provides a solution by iteration with rapid convergence towards the solution. This requires determining the Jacobian matrix from the functions defined by the above expression:

$$
\begin{aligned}
& 2 \cdot k_{2} \cdot \cos B x_{2}+2 \cdot k_{3} \cdot \cos B x_{3}- \\
& 2 \cdot A \cdot\left(1+\cos ^{2} B x_{2}+\cos ^{2} B x_{3}\right)=f_{1} \\
& 2 \cdot A \cdot\left[k_{2} \cdot x_{2} \cdot \operatorname{sen} B x_{2}+k_{3} \cdot x_{3} \cdot \operatorname{sen} B x_{3}\right]- \\
& A^{2} \cdot\left(x_{2} \cdot \operatorname{sen} 2 B x_{2}+x_{3} \cdot \operatorname{sen} 2 B x_{3}\right)=f_{2}
\end{aligned}
$$

In this way:

$$
\frac{\partial f_{1}}{\partial A}=-2 \cdot\left(1+\cos ^{2} B x_{2}+\cos ^{2} B x_{3}\right)
$$

Figure 3. Swell depending on trajectory.

Derivating and equating to zero:

$$
\begin{aligned}
& \frac{\partial M}{\partial A}=2 \cdot\left(z_{i}-z_{1}-A \cdot \cos B t\right) \cdot \cos B t=0 \\
& \frac{\partial M}{\partial B}=2 \cdot\left(z_{i}-z_{1}-A \cdot \cos B t\right) \cdot A \cdot t \cdot \operatorname{sen} B t=0
\end{aligned}
$$

Applying both expressions for the data referred in the approximation you can obtain these equations:

$\left.2 \cdot\left(z_{2}-z_{1}\right) \cdot \cos B t_{2}+2 \cdot\left(z_{3}-z_{1}\right) \cdot \cos B t_{3}\right)-$

$-2 \cdot A \cdot\left(\cos ^{2} B t_{1}+\cos ^{2} B t_{2}+\cos ^{2} B t_{3}\right)=0$

$2 \cdot A \cdot\left[\left(z_{2}-z_{1}\right) \cdot t_{2} \cdot \operatorname{sen} B t_{2}+\left(z_{3}-z_{1}\right) \cdot t_{3} \cdot \operatorname{sen} B t_{3}\right]-$

$-A^{2} \cdot\left(t_{1} \cdot \operatorname{sen} 2 B t_{1}+t_{2} \cdot \operatorname{sen} 2 B t_{2}+t_{3} \cdot \operatorname{sen} 2 B t_{3}\right)=0$

Making the following change of variable:

$$
\begin{aligned}
& k_{i}=z_{i}-z_{1} \\
& x_{i}=t_{i}-t_{1}
\end{aligned}
$$

The expressions are simplified:

$$
\begin{aligned}
& 2 \cdot k_{2} \cdot \cos B x_{2}+2 \cdot k_{3} \cdot \cos B x_{3}- \\
& -2 \cdot A \cdot\left(1+\cos ^{2} B x_{2}+\cos ^{2} B x_{3}\right)=0 \\
& 2 \cdot A \cdot\left[k_{2} \cdot x_{2} \cdot \operatorname{sen} B x_{2}+k_{3} \cdot x_{3} \cdot \operatorname{sen} B x_{3}\right]- \\
& -A^{2} \cdot\left(x_{2} \cdot \operatorname{sen} 2 B x_{2}+x_{3} \cdot \operatorname{sen} 2 B x_{3}\right)=0
\end{aligned}
$$$$
\begin{aligned}
& \frac{\partial f_{1}}{\partial B}=-2 \cdot\left(k_{2} \cdot x_{2} \cdot \operatorname{sen} B x_{2}+k_{3} \cdot x_{3} \cdot \operatorname{sen} B x_{3}\right)+ \\
& +2 \cdot A \cdot\left(x_{2} \cdot \operatorname{sen} 2 B x_{2}+x_{3} \cdot \operatorname{sen} 2 B x_{3}\right)
\end{aligned}
$$

$\frac{\partial f_{2}}{\partial A}=2 \cdot\left(k_{2} \cdot x_{2} \cdot \operatorname{sen} B x_{2}+k_{3} \cdot x_{3} \cdot \operatorname{sen} B x_{3}\right)-$

$-2 \cdot A \cdot\left(x_{2} \cdot \operatorname{sen} 2 B x_{2}+x_{3} \cdot \operatorname{sen} 2 B x_{3}\right)$

$\frac{\partial f_{2}}{\partial B}=2 \cdot A \cdot\left(k_{2} \cdot x_{2}^{2} \cdot \cos B x_{2}+k_{3} \cdot x_{3}^{2} \cdot \cos B x_{3}\right)-$

$-2 \cdot A^{2} \cdot\left(x_{2}^{2} \cdot \cos 2 B x_{2}+x_{3}^{2} \cdot \cos 2 B x_{3}\right)$

Therefore the Jacobian matrix is:

$$
J=\left[\begin{array}{ll}
\frac{\partial f_{1}}{\partial A} & \frac{\partial f_{1}}{\partial B} \\
\frac{\partial f_{2}}{\partial A} & \frac{\partial f_{2}}{\partial B}
\end{array}\right]
$$

Calling:

$$
F=\left[\begin{array}{l}
f_{1} \\
f_{2}
\end{array}\right]
$$


Proc. of the Fourth International Conference on Advances in Civil, Structural and Environmental Engineering - ACSEE 2016. Copyright (C) Institute of Research Engineers and Doctors. All rights reserved.

ISBN: 978-1-63248-114-6 doi: 10.15224/ 978-1-63248-114-6-22

Newton's method proposes the iterative resolution of the linear system of equations:

$$
\left[\begin{array}{l}
A^{k} \\
B^{k}
\end{array}\right]=\left[\begin{array}{l}
A^{k-1} \\
B^{k-1}
\end{array}\right]-\left(J^{k-1} \cdot F^{k-1}\right)
$$

That is to say:

$$
J^{k-1} \cdot\left[\begin{array}{c}
A^{k}-A^{k-1} \\
B^{k}-B^{k-1}
\end{array}\right]=-F^{k-1}
$$

Calling:

$$
\begin{gathered}
a=1+\cos ^{2} B^{k-1} x_{2}+\cos ^{2} B^{k-1} x_{3} \\
b=x_{2} \cdot \operatorname{sen} 2 B^{k-1} x_{2}+x_{3} \cdot \operatorname{sen} 2 B^{k-1} x_{3} \\
c=2 \cdot\left(k_{2} \cdot x_{2} \cdot \operatorname{sen} B^{k-1} x_{2}+z_{3} \cdot k_{3} \cdot \operatorname{sen} B^{k-1} x_{3}\right) \\
d=2 \cdot\left(k_{2} \cdot \cos B^{k-1} x_{2}+k_{3} \cdot \cos B^{k-1} x_{3}\right) \\
e=\left(\frac{\partial f_{2}}{\partial B}\right)^{k-1}=2 A^{k-1}\left(k_{2} \cdot x_{2}^{2} \cdot \cos B^{k-1} x_{2}+\right. \\
\left.+k_{3} \cdot x_{3}^{2} \cdot \cos B^{k-1} x_{3}\right)-2 A^{k-12}\left(x_{2}^{2} \cdot \cos 2 B^{k-1} x_{2}+x_{3}^{2} \cdot \cos 2 B^{k-1} x_{3}\right)
\end{gathered}
$$

The system of equiations is:

$$
\left[\begin{array}{cc}
-2 \cdot a & -c+2 \cdot A^{k-1} \cdot b \\
c-2 \cdot A^{k-1} \cdot b & e
\end{array}\right] \cdot\left[\begin{array}{l}
A^{k}-A^{k-1} \\
B^{k}-B^{k-1}
\end{array}\right]=\left[\begin{array}{c}
d-2 \cdot A^{k-1} \cdot a \\
A^{k-1} \cdot c-A^{k-1^{2}} \cdot b
\end{array}\right]
$$

Calculating:

$$
\begin{aligned}
& {\left[\begin{array}{l}
A^{k} \\
B^{k}
\end{array}\right]=\frac{1}{\left(c-2 \cdot A^{k-1} \cdot b\right)^{2}-2 \cdot a \cdot e} \cdot\left[\begin{array}{cc}
e & -c+2 \cdot A^{k-1} \cdot b \\
c-2 \cdot A^{k-1} \cdot b & -2 \cdot a
\end{array}\right] .} \\
& \cdot\left[\begin{array}{c}
2 \cdot A^{k-1} \cdot B^{k-1} \cdot b-d-c \cdot B^{k-1} \\
e \cdot B^{k-1}-A^{k-1^{2}} \cdot b
\end{array}\right]
\end{aligned}
$$

The first value for variables $\mathrm{A}$ and $\mathrm{B}$ are recommended to be: $\mathrm{A}=1 \mathrm{~m}, \mathrm{~B}=1 / 2 \pi$ corresponding to a wave amplitude of one meter and a period of one second because with these initial values the solution converges quickly for common situations.

\section{B. Mathematical model for correction of depth measurements.}

Once modeled vertical movement of the boat according to equation (2): $z=F(t)=z_{i}+A \cdot \cos B t$; for the time of depth reading $t_{2}$, the depth read by the echo sounder $p_{2}$ should be corrected by the travel time of the signal, effect of the vertical movement of the vessel.

Thus the instant output pulse of the sounder corresponds to the instant:

$$
t_{i}=t_{2}-\delta t
$$

The value $\delta t$ can be calculated with sufficient accuracy as the time it takes the pulse emitted by the transducer to return to him:

$$
\delta t=\frac{p_{2}}{v_{c}}
$$

The height of the boat for measurement moments $t_{2}$ and $t_{i}=t_{2}-\delta t$ have these values:

$$
\begin{gathered}
F_{2}=z_{1}+A \cdot \cos B t_{2} \\
F_{i}=z_{1}+A \cdot \cos B t_{i}
\end{gathered}
$$

Since the boat has undergone a vertical movement during measurement, the correction will be:

$$
\Delta p=F_{2}-F_{i}=A \cdot\left(\cos B t_{2}-\cos B t_{i}\right)
$$

Therefore, the depth corrected by the effecto of the vertical movement has a value of:

$$
p_{c a}=p_{m}+\Delta p
$$

\section{Results and conclusions.}

From the proposed model, it is possible to affirm that a sine-wave model to reflect the vertical movement is the most appropriate given their relative simplicity of calculation and correspondence with the physical reality of the movement, variable depending on both the swell and the relationship of this one with the boat.

Depth reading correction depends largely on the depth obtained. That is, since the speed of sound in water is 
Proc. of the Fourth International Conference on Advances in Civil, Structural and Environmental Engineering - ACSEE 2016. Copyright $(\odot$ Institute of Research Engineers and Doctors. All rights reserved.

ISBN: 978-1-63248-114-6 doi: 10.15224/ 978-1-63248-114-6-22

relatively high (about $1500 \mathrm{~m} / \mathrm{s}$ ) and the depths obtained by the echo sounder are usually of low magnitude in applications of civil engineering, the duration of a measure is very small and therefore, the vertical movement of the boat as well.

Therefore, one can ensure that the incidence of movement studied in small depths (up to $30 \mathrm{~m}$ ) can be neglected. Not so for great depths, where their value can approach even the full amplitude of motion.

One possible approach to the value of this correction is presented in the table and graph below, calculated from a vertical variable speed boat, a maximum depth of $30 \mathrm{~m}$ and a speed of sound in water of $1500 \mathrm{~m} / \mathrm{s}$.

TABLE I. CORRECTION DUE TO VERTICAL MOVEMENT. (CM)

\begin{tabular}{|c|r|r|r|r|r||}
\cline { 2 - 6 } \multicolumn{1}{c|}{} & \multicolumn{5}{c|}{ Vertical speed (m/s) } \\
\hline Depth(m) & 0.4 & 0.6 & 0.8 & 1 & 1.5 \\
\hline \hline 5 & 0.3 & 0.4 & 0.5 & 0.7 & 1.0 \\
\hline 10 & 0.5 & 0.8 & 1.1 & 1.3 & 2.0 \\
\hline 15 & 0.8 & 1.2 & 1.6 & 2.0 & 3.0 \\
\hline 20 & 1.1 & 1.6 & 2.1 & 2.7 & 4.0 \\
\hline 25 & 1.3 & 2.0 & 2.7 & 3.3 & 5.0 \\
\hline 30 & 1.6 & 2.4 & 3.2 & 4.0 & 6.0 \\
\hline
\end{tabular}

In view of the results obtained it can be concluded that for depths up to 30 meters can reach neglected the effect of vertical movement, especially in inland areas where the swell is very small.

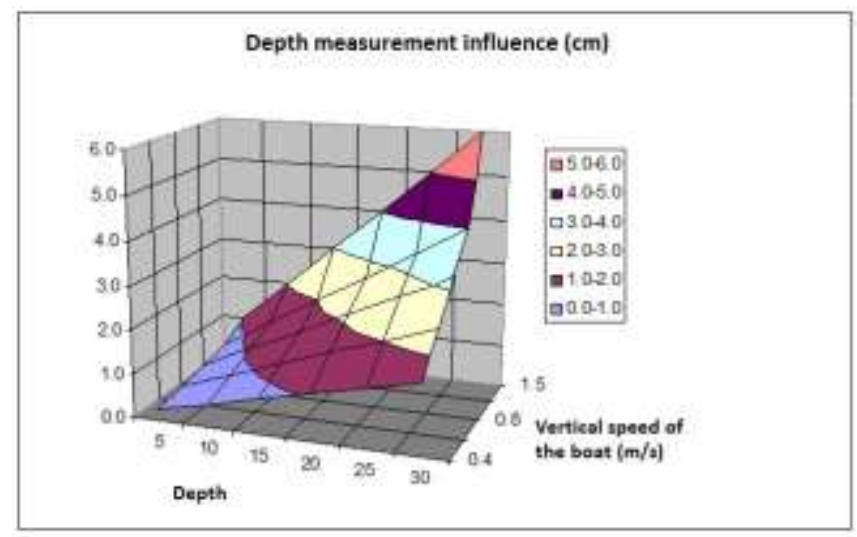

Figure 4. Depth measurement influence of vertical speed $(\mathrm{cm})$.

In any case, it is recommended not to run bathymetric surveying campaigns when swell conditions are unfavorable, with speeds of ascent or descent of the upper vessel at $1 \mathrm{~m} / \mathrm{s}$, provided the required accuracy so dictates.

\section{References}

[1] W. Maleika, "The influence of track configuration and multibeam echosounder parameteres on the accuracy of seabed DTMs obtained in shallow water". Earth Science Informatics 6(2): 139-159. Doi 10.1007/s12145-013.0111-9

[2] Abramowitz, M., and I.A. Stegun. 1972. Handbook of Mathematical Functions. New York: Dover Publications.

[3] American Society of Civil Engineers (ASCE). 1998. Hydrographic Surveying (Technical Engineering and Design Guides as Adapted from the U.S. Army Corps of Engineers, No. 25). Reston: ASCE.

[4] U.S. Army Corps of Engineers (USACE). 2002. Engineering and Design: Hydrographic Surveying (Engineer Manual 1110-2-1003), Washigton DC: USACE.

[5] Pereda R, et al; "Model for the processing an estimation of dual frequency sounder observations in detailed bathymetries". Marine Geodesy, Vol 392016 Pg 305-320

[6] MacQueen, J. B. 1967. Some methods for classification an analysis of multivariate observations. In Proceedings of the Berkeley Symposium on Mathematics Statistics and Probability, Vol.1., eds. L.M. Le Cam, and J. Neyman, 281-297. Berkley: University of California Press.

[7] Hughes Clarke, J. 2000. Present-day Methods of Depth Measurement. In Continental Shelf Limits: The Scientific and Legal Interface, eds. P. Cook, and C. Carlton, 139-159. New York: Oxford University Press.

[8] Hellequin, L. 1998. Statistical characterization of multibeam echosounder data. Paper, Oceans 1998, Nice, September 28 - October 1.

[9] Eleftherakis, D., A. Amiri-Simkooei, M. Snellen, and D.G. Simons. 2012. Improving riverbed sediment classification using backscatter and depth residual features of multi-beam echo-sounder systems. Journal of the Acoustical Society of America 131(5):3710-3725. doi: 10.1121/1.3699206. 